\title{
COMMUTATORS IN GROUPS OF ORDER-PRESERVING PERMUTATIONS
}

\author{
by MANFRED DROSTE and R. M. SHORTT
}

(Received 12 July, 1989)

1. Introduction. Let $(S, \leq)$ be a poset (partially ordered set), $A(S)=\operatorname{Aut}(S, \leq)$ its automorphism group and $G \subseteq A(S)$ a subgroup. In the literature, various authors have studied sufficient conditions on $G$ and the structure of $(S, \leq)$ which imply that $G$ is simple or perfect. Let us call $(S, \leq)$ doubly homogeneous if each isomorphism between two 2-subsets of $S$ extends to an isomorphism of $(S, \leq)$. Higman [8] proved that if $(S, \leq)$ is a doubly homogeneous chain then $B(S)$, the group of all automorphisms of $(S, \leq)$ with bounded support, is simple, and each element of $B(S)$ is a commutator in $B(S)$. Droste, Holland and Macpherson [5] showed that if $(S, \leq)$ is a doubly homogeneous tree then its automorphism group again contains a unique simple normal subgroup in which each element is a commutator. Dlab [3] established similar results for various groups of locally linear automorphisms of the reals. Further results in this direction are contained in Glass [7]. It is the aim of this note to establish a common generalization and sharpening of the previously mentioned results.

Let us introduce some notation. For any poset $(S, \leq)$ and $a, b \in S$ with $a<b$, set $\langle a, b\rangle=\{s \in S: a \leq s, b \nless s\}$, an interval in $S$. If $f \in A(S)$, put $\operatorname{supp}(f)=\left\{s \in S: s \neq s^{f}\right\}$, the support of $f$. We say that $f$ has bounded support if there are $a, b \in S$ with $a<b$ and $\operatorname{supp}(f) \subseteq\langle a, b\rangle$. Let $B(S)$ be the set of all automorphisms of $S$ with bounded support. We note that, in most cases considered here, $B(S)$ is a subgroup of $A(S)$, although this is not true in general. Now let $G, H$ be subsets of $A(S)$ with $G \subseteq H$. We say that $H$ is closed under $\omega$-patching of conjugate elements of $G$ if, whenever $a_{i}, b_{i}, c_{i} \in S, g_{i} \in G(i \in \mathbb{N})$ and $h \in H$ such that $a_{i}<b_{i}<c_{i}<a_{i+1}, \operatorname{supp}\left(g_{i}\right) \subseteq\left\langle b_{i}, c_{i}\right\rangle$ and $g_{i}=h^{-i} g_{0} h^{i}$ for each $i \in \mathbb{N}$, the mapping $k: S \rightarrow S$ defined by $\left.k\right|_{\left\langle a_{i}, a_{i+1}\right\rangle}=g_{i}(i \in \mathbb{N})$ and $\left.k\right|_{s \cup \cup_{i \in N}\left\langle a_{i}, a_{i+1}\right\rangle}=$ id belongs to $H$. (Observe that this condition is always satisfied, for instance, if $(S, \leq)$ is a chain and $H=B(S)$ or $H=A(S)$.) Finally, we say that $G \subseteq A(S)$ is feebly 1-transitive if, for any $a, b \in S$ with $a<b$, there is $g \in G$ with $b \leq a^{g}$, and $G$ is feebly 2-transitive if, for any $a, b, c, d \in S$ with $a<b, c<d$, there exists $g \in G$ with $a \leq c^{g}<d^{g} \leq b$. We will show the following result.

Theorem 1.1. Let $(S, \leq)$ be an infinite chain, $H$ a subgroup of $A(S)$, and $G=H \cap B(S)$. Assume that $H$ is closed under $\omega$-patching of conjugate elements of $G$.

(a) If $H$ is feebly 1-transitive then each element of $G$ is a commutator in $H$.

(b) If $G$ is feebly 1-transitive then each element of $G$ is a commutator in $G$.

(c) If $H$ is feebly 2-transitive then, for any $g \in G$ and $h \in H$ with $h \neq 1$, there are $k_{1}, k_{2} \in H$ such that $g=h^{k_{1}}\left(h^{-1}\right)^{k_{2}}$. In particular, $G$ is contained in every non-trivial normal subgroup of $H$.

(d) If $G$ is feebly 2-transitive then, for any $g, h \in G$ with $h \neq 1$, there are $k_{1}, k_{2} \in G$ such that $g=h^{k_{1}}\left(h^{-1}\right)^{k_{2}}$. In particular, $G$ is simple.

As mentioned before, Theorem 1.1 generalizes results of $[3,5,7,8]$. Applied to the group $H$ comprising all $h \in A(\mathbb{R})$ such that $h$ and $h^{-1}$ are right differentiable, with $G=H \cap B(\mathbb{R})$, it sharpens McCleary [10, Theorem 8]. With a similar argument as for Theorem 1.1, we obtain the following sharpening of [10, Theorem 5].

Glasgow Math. J. 33 (1991) 55-59. 
Corollary 1.2. Let $G$ be the group of all diffeomorphisms of $\mathbb{R}$ with bounded support. Then each element of $G$ is a commutator in $G$, and, whenever $g, h \in G$ with $h \neq 1$, there are $k_{i} \in G(i=1, \ldots, 4)$ such that $g=h^{k_{1}} \cdot\left(h^{-1}\right)^{k_{2}} \cdot h^{k_{3}} \cdot\left(h^{-1}\right)^{k_{4}}$. In particular, $G$ is simple.

Here, the second part of Corollary 1.2 is immediate from the first part and a lemma of Higman [8].

A poset $(T, \leq)$ containing an infinite chain and at least two incomparable elements is called a tree if any two elements of $T$ have a common lower bound, but no two incomparable elements of $T$ have a common upper bound. Doubly homogeneous trees and their automorphism groups have been studied in [4]-[6], [9]; they also occur in a recent classification result of Adeleke and Neumann [1] for certain Jordan groups. If $T$ is a tree, let

$$
S(T)=\left\{g \in A(T):(\exists x \in T)(\forall y \in T)\left(y^{g} \neq y \Rightarrow x<y\right)\right\},
$$

a normal subgroup of $A(T)$. A segment of $T$ is a convex subchain $C \subseteq T$ such that, whenever $z \in C$ and $x \in T \backslash C$ with $z<x, c<x$ for each $c \in C$. Thus a segment is a convex chain with no branches growing from its sides. A chain is rigid if it has no non-trivial automorphism. The following result sharpens [5, Theorem 1.1].

THEOREM 1.3. Let $(T, \leq)$ be a tree such that $S(T)$ is feebly 1-transitive. Then each element of $S(T)$ is a commutator in $S(T)$, and the following are equivalent:

(1) $S(T)$ is simple;

(2) $S(T)$ is contained in every non-trivial normal subgroup of $A(T)$;

(3) each segment of $T$ is rigid.

2. Proof of the results. Our argument uses a technique of Anderson [2] which allows one, under certain conditions, to write elements of permutation groups as commutators. We also employ a lemma of Higman [8] for permutation groups which gives a sufficient condition for the commutator subgroup to be simple. Anderson's technique is used to prove the following proposition.

Proposition 2.1. Let $(S, \leq)$ be a poset containing an infinite chain, $H$ a subgroup of $A(S)$, and $G=H \cap B(S)$. Assume that $H$ is closed in $A(S)$ under $\omega$-patching of conjugate elements of $G$.

(a) If $H$ is feebly 1-transitive then each element of $G$ is a commutator in $H$.

(b) If $G$ is feebly 1-transitive then each element of $G$ is a commutator in $G$.

(c) Let $H$ be feebly 2-transitive. Then, for any $g \in G$ and $h \in H$ such that $s<s^{h}$ for some $s \in S$, there are $k_{1}, k_{2} \in H$ such that $g=\left(h^{-1}\right)^{k_{1}} h^{k_{2}}$. Moreover, if $G$ is feebly 2-transitive and $h \in G$ then $k_{1}, k_{2}$ can be chosen from $G$.

Proof. Let $g \in G$. As $H$ is feebly 1 -transitive, there are $a, b, c, d \in S$ such that $a<b<c<d$ and $\operatorname{supp}(g) \subseteq\langle b, c\rangle$; also, there is $h \in H$ with $d \leq a^{h}$. Now put $a_{i}=a^{h^{i}}$ $(i \in \mathbb{N})$. Then $a_{i}<a_{i+1}$ for each $i \in \mathbb{N}$. Define $k: S \rightarrow S$ by putting

$$
x^{k}= \begin{cases}x^{h^{-i} \cdot g \cdot h^{i}} & \text { if } \quad x \in\left\langle a_{i}, a_{i+1}\right\rangle(i \in \mathbb{N}), \\ x & \text { if } \quad x \in S \backslash \bigcup_{1 \in \mathbb{N}}\left\langle a_{i}, a_{i+1}\right\rangle .\end{cases}
$$


Then $k \in H$ and $g=k \cdot h^{-1} \cdot k^{-1} \cdot h$, which proves (a). Observe here that if $h \in G$ then also $k \in H \cap B(S)=G$. This implies (b).

Now let $H$ be feebly 2-transitive and $h \in H, s \in S$ with $s<s^{h}$. For any $x, y \in S$ with $x<y$, there is $k \in H$ (even $k \in G$, if $G$ is feebly 2-transitive) such that $s^{k} \leq x<y \leq s^{h k}$; thus $y \leq x^{k^{-1} \cdot h . k}$. Together with the above argument, this implies (c).

Proof of Theorem 1.1. This is now immediate by Proposition 2.1.

Let $(A, \leq),(B, \leq)$ be two posets and $S=A \times B$. We say $(S, \leq)=(A, \leq) \times(B, \leq)$ is ordered lexicographically if, for any $a, a^{\prime} \in A$, and $b, b^{\prime} \in B$, we have that $(a, b) \leq\left(a^{\prime}, b^{\prime}\right)$ in $S$ if and only if either $a<a^{\prime}$ or $a=a^{\prime}, b \leq b^{\prime}$. Hence $(S, \leq)$ is ordered as $(A, \leq)$ copies of $(B, \leq)$. An infinite chain $(C, \leq)$ is called $k$-homogeneous (where $k \in \mathbb{N}$ ) if, for any two subsets $A, B \subseteq C$ with $|A|=|B|=k$, there exists $g \in A(C)$ with $A^{g}=B$. Now let $(C, \leq)$ be $k$-homogeneous for some $k \geq 2$. Then, as is well known (cf., e.g., $[7, \S 1.10])$, for any two subsets $A, B \subseteq C$ with $|A|=|B| \in \mathbb{N}$, there exists $g \in B(C)$ with $A^{g}=B$. As an immediate consequence of this remark and of Theorem 1.1, we obtain the following corollary.

Corollary 2.2. Let $(C, \leq)$ be a 1-homogeneous chain, let $(P, \leq)$ be any poset, and let $(S, \leq)=(C, \leq) \times(P, \leq)$ be ordered lexicographically. Then each element of $B(S)$ is a commutator in $A(S)$. If , moreover, $(C, \leq)$ is 2-homogeneous then each element of $B(S)$ is a commutator in $B(S)$.

As an example for Corollary 2.2 , let $(S, \leq)=(C, \leq) \times(P, \leq)$, where first $(C, \leq)=$ $(\mathbb{Z}, \leq)$ and either $(P, \leq)=(\mathbb{Z}, \leq)$ or $(P, \leq)$ is an antichain with at least two elements. Then $(S, \leq)$ is 1 -homogeneous, $B(S)$ properly contains its commutator subgroup, but each element of $B(S)$ is a commutator in $A(S)$. Secondly, let $(C, \leq)=(\mathbb{Q}, \leq)$ and let $(P, \leq)$ be any poset. Then each element of $B(S)$ is a commutator in $B(S)$. Next we turn to the argument for Corollary 1.2 and Theorem 1.3. Since in Corollary 1.2 the group $G$ of all diffeomorphisms of $\mathbb{R}$ with bounded support is not closed under $\omega$-patching of conjugate elements of $G$, we will need the following lemma.

Lemma 2.3 (Higman [8]). Let $H$ be a permutation group on a set $S$, and let $G \subseteq H$. Assume that, for any $f, g \in G$ and $h \in H$ with $h \neq 1$, there is $k \in G$ with $A^{k} \cap A^{k h}=\varnothing$, where $A=\operatorname{supp}(f) \cup \operatorname{supp}(g)$. Then $[G, G]$ is simple and contained in every non-trivial normal subgroup of $H$.

Proof (sketch). Given $f, g \in G$ and $h \in H$ with $h \neq 1$, choose $k \in G$ as indicated, and put $k_{1}=k^{-1} \cdot f, k_{2}=k^{-1}, k_{3}=k^{-1} \cdot g, k_{4}=k^{-1} \cdot f \cdot g \in G$. Observing that $\left(g^{-1}\right)^{k}$ and $f^{k h}$ commute, we obtain $[f, g]=\left(h^{-1}\right)^{k_{1}} \cdot h^{k_{2}} \cdot\left(h^{-1}\right)^{k_{3}} \cdot h^{k_{4}}$. This implies the result.

Using a similar argument as for Proposition 2.1, we now prove Corollary 1.2.

Proof of Corollary 1.2. (Here we let functions operate from the left on the argument.) By Lemma 2.3, it suffices to show that each element of $G$ is a commutator in $G$. Let $g \in G$. Choose $a, b, c, d \in \mathbb{R}$ with $a<b<c<d$ and $\operatorname{supp}(g) \subseteq\langle b, c\rangle$. Next choose $h \in G$ such that $d \leq h(a)$. If we put $a_{i}=h^{i}(a)(i \in \mathbb{N})$ and $z=\lim _{i \rightarrow \infty} a_{i} \in \mathbb{R}$ then $h^{\prime}(z)=1$. Note that $h(z)=z$ and $\left(h^{-1}\right)^{\prime}(z)=1$. Define $k: \mathbb{R} \rightarrow \mathbb{R}$ by putting $k(x)=h^{i} \circ g \circ h^{-i}(x)$ if 
$x \in\left\langle a_{i}, a_{i+1}\right\rangle$ for some $i \in \mathbb{N}$, and $k(x)=x$ otherwise. Now let $x \in \mathbb{R}$ with $a \leq x<z$. As

$$
\frac{h(x)-h(z)}{x-z} \leq \frac{k(x)-k(z)}{x-z} \leq \frac{h^{-1}(x)-h^{-1}(z)}{x-z},
$$

it follows that $k$ is differentiable at $z$ and $k^{\prime}(z)=1$. Hence $k \in G$, and $g=h \circ k^{-1} \circ h^{-1} \circ k$.

Next we prove Theorem 1.3. If $(T, \leq)$ is a tree and $y, z \in T$, we write $y \| z$ to denote that $y$ and $z$ are incomparable, i.e. neither $y \leq z$ nor $z \leq y$. If $A \subseteq T$, let $z<A$ indicate that $z<a$ for each $a \in A$.

Proof of Theorem 1.3. First note that, since $S(T)$ is feebly 1-transitive, for any $z \in T$, there are $x, y \in T$ such that $x<\{y, z\}$ and $y \| z$, and thus $\{t \in T: z \leq t\} \subseteq\langle x, y\rangle$. Hence $S(T)=B(T)$; also $S(T)$ is closed under $\omega$-patching of conjugate elements of $S(T)$. By Proposition 2.1, each element of $S(T)$ is a commutator in $S(T)$.

$(1) \rightarrow(3)$ and (2) $\rightarrow(3)$. Assume $C$ is a non-rigid segment of $T$. Choose $g \in A(T)$ with $g \neq 1$ and $\operatorname{supp}(g) \subseteq C$. Let $c \in C$. There are $x, y \in T$ such that $x<\{c, y\}$ and $c \| y$. Thus $x<C$ and $g \in S(T)$. Next note that the union of any two segments of $T$ with non-trivial intersection is again a segment. Hence, if $h \in A(T)$ is any product of conjugates of $g$ or $g^{-1}$ then, for each $t \in T$, either $t \leq t^{h}$ or $t^{h} \leq t$. Now choose $f \in S(T)$ with $c^{f} \leq x$. Then $y \| y^{f}$. Thus $f$ does not belong to the normal subgroup generated by $g$ in $A(T)$, a contradiction.

$(3) \rightarrow(1)$ and (3) $\rightarrow(2)$. Let $h \in A(T)$ with $h \neq 1$. We claim there is $t \in T$ with $t \| t^{h}$. Choose $a \in T$ with $a \neq a^{h}$. We may assume that $a<a^{h}$ or $a^{h}<a$. Let $C$ be the convexification of the chain $\left\{a^{h^{i}}: i \in \mathbb{Z}\right\}$ in $T$. As $C$ cannot be a segment of $T$, there are $x, y \in C$ and $z \in T$ with $x<\{y, z\}$ and $y \| z$. Then $z \| z^{h}$, and we put $t=z$.

Now let $f, g \in S(T)$ and put $A=\operatorname{supp}(f) \cup \operatorname{supp}(g)$. Let $w \in T$ with $w<A$. There is $k \in S(T)$ with $t^{k}<w$; hence $w \| w^{k^{-1} h k}$ and $A \cap A^{k^{-1} h k}=\varnothing$. Since each element of $S(T)$ is a commutator in $S(T)$, Lemma 2.3 implies the result.

We note here that, as the argument shows, under assumption (3) of Theorem 1.3, for any $g \in S(T)$ and $h \in A(T)$ with $h \neq 1$, there are $k_{i} \in S(T)(i=1, \ldots, 4)$ such that $g=h^{k_{1}} \cdot\left(h^{-1}\right)^{k_{2}} \cdot h^{k_{3}} \cdot\left(h^{-1}\right)^{k_{4}}$, which sharpens assertions (1) and (2).

We conclude with some remarks to Theorem 1.3. A tree $(T, \leq)$ is called weakly 2-transitive if, for any $a, b, c, d \in T$ with $a<b$ and $c<d$, there exists $g \in A(T)$ with $a^{g}=c$ and $b^{g}=d$. In this case, for any $a, b, c, d \in T$ with $a<b$ and $c<d$ there is also $g \in S(T)$ with $a^{g}=c$ and $b^{g}=d$ (cf. [5, Theorem 3.3]). Now let $(T, \leq)$ be a weakly 2-transitive tree, $(C, \leq)$ any chain, and $\left(T^{*}, \leq\right)=(T, \leq) \times(C, \leq)$, ordered lexicographically. Then $\left(T^{*}, \leq\right)$ is a tree, and, by the preceding remark, $S\left(T^{*}\right)$ is feebly 1-transitive. Hence, by Theorem 1.3, each element of $S\left(T^{*}\right)$ is a commutator in $S\left(T^{*}\right)$, and $S\left(T^{*}\right)$ is simple if and only if $(C, \leq)$ is rigid, since the segments of $T^{*}$ are precisely the copies of $C$.

Acknowledgement. This work was done while the first-named author was visiting Wesleyan University in March 1989. He would like to thank his colleagues at the Department of Mathematics for their hospitality and an enjoyable stay.

\section{REFERENCES}

1. S. Adeleke and P. M. Neumann, On infinite Jordan groups, manuscript, 1987.

2. R. D. Anderson, The algebraic simplicity of certain groups of homeomorphisms, Amer. J. Math. 80 (1958), 955-963. 
3. V. Dlab, On a family of simple ordered groups, J. Austral. Math. Soc. 8 (1968), 591-608.

4. M. Droste, Structure of partially ordered sets with transitive automorphism groups, Memoirs Amer. Math. Soc. 334 (1985).

5. M. Droste, W. C. Holland and H. D. Macpherson, Automorphism groups of infinite semilinear orders (I), Proc. London Math. Soc. (3) 58 (1989), 454-478.

6. M. Droste, W. C. Holland and H. D. Macpherson, Automorphism groups of infinite semilinear orders (II), Proc. London Math. Soc. (3) 58 (1989), 479-494.

7. A. M. W. Glass, Ordered permutation groups, London Math. Soc. Lecture Note Series 55 (Cambridge University Press, 1981).

8. G. Higman, On infinite simple permutation groups, Publ. Math. Debrecen 3 (1954), 221-226.

9. J. Maroli, Tree permutation groups (Ph.D. thesis, Bowling Green State University, Bowling Green, Ohio, 1989).

10. S. H. McCleary, Some simple homeomorphism groups having nonsolvable outer automorphism groups, Comm. Algebra 6 (1978), 483-496.

FACHBEREICH 6-MATHEMATIK

UNIVERSITÄT GHS ESSEN

4300 ESSEN 1

Germany
Department of Mathematics

WESLEYAN UNIVERSITY

MidDLETOWN

CONNECTICUT 06457

U.S.A. 